\title{
Reading of Capsule Endoscopy Images Significantly Improved
}

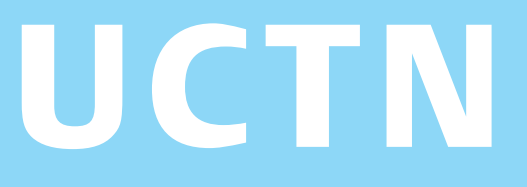

Capsule endoscopy (Given Imaging, Yoqneam, Israel) allows for small-bowel assessment with little discomfort for the patient. However, evaluation is still a challenge for the investigator. Unlike flexible endoscopy where the examiner is actively involved in maneuvering the instruments, browsing more than 50000 images in capsule endoscopy can be a monotonous procedure. We added a "jog/ shuttle" control, which is a well-established tool in professional video editing, to the Given Imaging capsule endoscopy reading system.

The jog/shuttle control (Contour Design, Inc. Windham, New Hampshire, USA) was connected to the USB port. The inner ring or "jog" rotates through $360^{\circ}$ and provides precision frame-by-frame control. The outer ring or "shuttle" is rubberized and spring-loaded, and was configured to control browsing speed; seven different frame speeds could be obtained, according to the torque applied. Turning right or left moved the video forward and backward. Additional buttons were configured as shown in Figure 1.

Volunteer doctors evaluated two training videos for 5:30 minutes each, with randomization of the order of the two videos and the order of evaluation with and without the jog/shuttle control.

Preliminary evaluation of the first 12 volunteers did not show improvement in the detection rate with use of the jog/shuttle control. However, overall, $25 \%$ more images were reviewed. In a questionnaire examiners reported that they found capsule endoscopy evaluation significantly easier with the jog/shuttle control $(P=0.005)$, they felt significantly more actively involved $(P=0.002)$, and preferred the addition of the jog/shuttle control significantly $(P=0.002)$.

Using the jog/shuttle control with the left hand and the mouse with the right hand (Figure 2) was found to be the most prac-

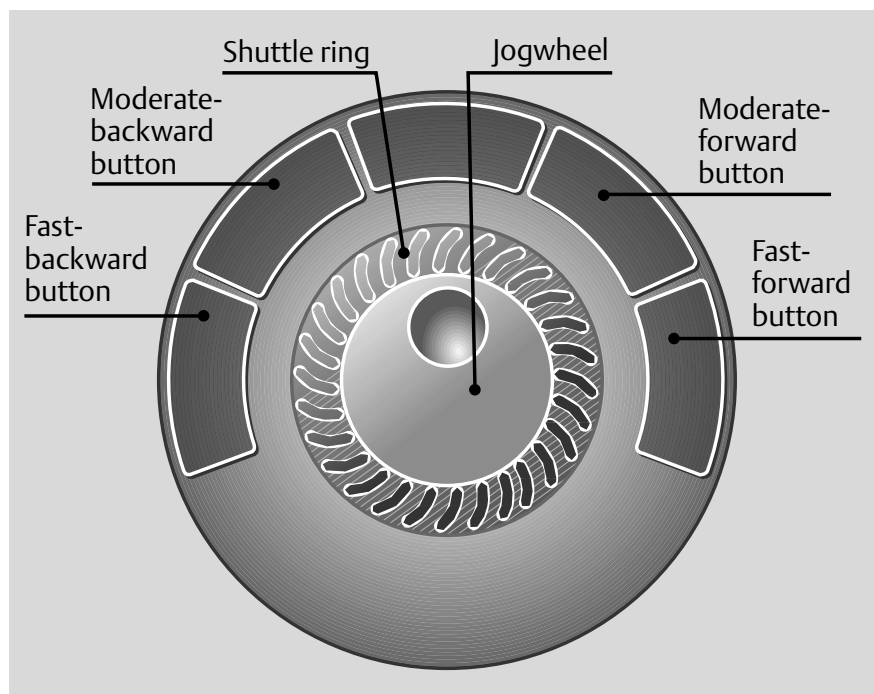

Figure 1 Configuration of the jog/shuttle control.

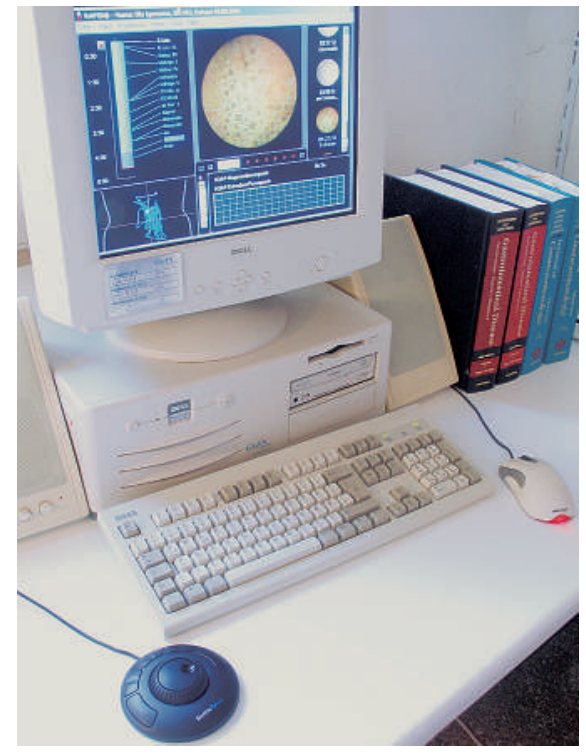

Figure 2 Placing the jog/shuttle control on the left and the mouse on the right side appeared to be the most practical solution.

tical solution in the evaluation of three complete cases.

Our preliminary results indicate an advantage in the use of a jog/shuttle control for capsule endoscopy evaluation.
U. Seitz, Y. M. Naga, S. Bohnacker, S. Seewald, N. Soehendra

Department of

Interdisciplinary Endoscopy, University Hospital Eppendorf, Hamburg, Germany

\section{Corresponding Author}

\section{U. Seitz, M.D.}

Department of Interdisciplinary Endoscopy University Hospital Hamburg-Eppendorf University of Hamburg

Martinistraße 52

20246 Hamburg

Germany

Fax: $\quad+49-40-428034420$

E-mail: seitz@uke.uni-hamburg.de 